\title{
Conceptual Studies for Exploiting the TerraSAR-X Dual Receive Antenna
}

\author{
Josef Mittermayer \\ Deutsches Zentrum für Luft- und Raumfahrt (DLR) \\ Institut für Hochfrequenztechnik \& Radarsysteme \\ 82234 Oberpfaffenhofen, Germany \\ T: +49-8153-28-2373, E-Mail: josef.mittermayer@dlr.de
}

\author{
Hartmut Runge \\ Deutsches Zentrum für Luft- und Raumfahrt (DLR) \\ Institut für Methoden der Fernerkundung \\ 82234 Oberpfaffenhofen, Germany \\ T: +49-8153-28-1364, E-Mail: hartmut.runge@dlr.de
}

\begin{abstract}
This Paper describes the newly introduced Dual Receive Antenna Mode (DRA) of the TerraSAR-X instrument. It explains the principle of forming two receiving antennas and deals with the already identified new applications and experiments enabled by this new mode, e.g. along track interferometry, geometric resolution enhancement and full polarimetric mode.
\end{abstract}

Keywords: TerraSAR-X, DRA-Mode, ATI, Stripmap Resolution Enhancement, Full Polarimetric Mode

\section{INTRODUCTION}

The German TerraSAR-X satellite [1], [2] is implemented in a Public-Private-Partnership between the German Aerospace Center (DLR) and the ASTRIUM GmbH. It is equipped with a highly flexible phased array antenna which allows for SAR Stripmap, ScanSAR and Spotlight operations. The new Dual Receive Antenna Mode (DRA) of TerraSAR-X again increases the number of possible modes and applications.

With the Shuttle Radar Topography Mission (SRTM) it has been demonstrated in the year 2000 how an existing SAR (SIR$\mathrm{C} / \mathrm{X}-\mathrm{SAR}$ ) can be upgraded with a secondary antenna and receiving channel. The SRTM was aimed for the generation of digital elevation models with Across Track Interferometry and a $60 \mathrm{~m}$ long mast was used to separate the two antennas [3]. Furthermore the along track separation of 7 meters between the two antennas has been used to demonstrate space-borne Along Track Interferometry (ATI). Measurements of tidal ocean currents have been compared with existing oceanographical models with excellent agreement [4], [5] and it has been demonstrated that the speed of car traffic can be measured from space agreement [6].

The DRA concept of TerraSAR-X now does without any mast or secondary antenna. It applies the group antenna concept and logically splits the long standard antenna during receive into two parts separated in along track. The extra effort for this upgrade was minimal because for the second channel an already existing redundant receiver chain has been used. On the other hand the return for additional applications is considerable. These are mainly:

Along Track Interferometry (ATI)

- $\quad$ Strip Map Resolution Enhancement

- Full Polarimetric Mode

\section{FORMATION OF TWO RECEIVE ANTENNAS}

In the DRA concept of TerraSAR-X the complete antenna is used for transmission but in receive the antenna is divided into two separate partitions in along track. The signals of both receiving antennas are detected and recorded separately. As shown in figure 1, the azimuth pattern of the two halved partitions are doubled in beamwidth compared to the complete antenna. It has also to be kept in mind that the $\mathrm{Rx}$ gain is reduced by $3 \mathrm{~dB}$ for a halved receive antenna.

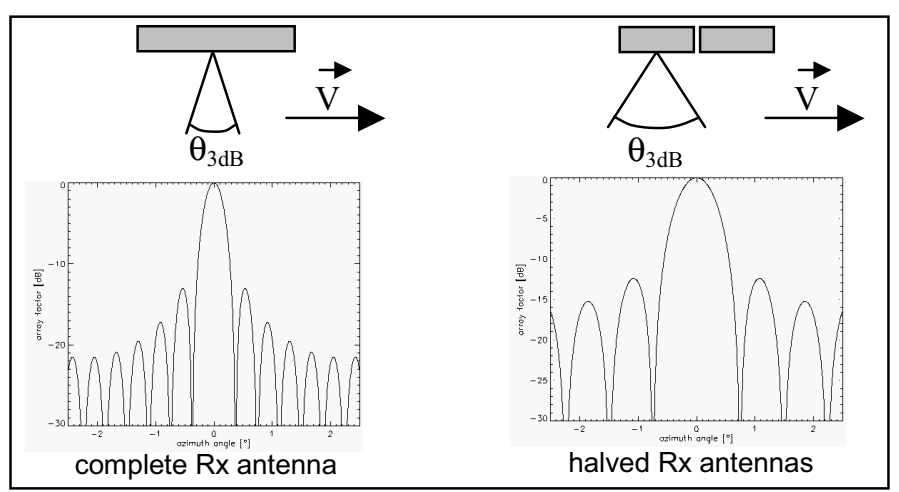

Figure 1. Azimuth pattern of complete (left) and halved (right) Rx antenna

Two options for the azimuth Tx pattern are considered in the following. In Configuration $\mathbf{A}$, it is assumed that by appropriate means, e.g. beam spoiling, the Tx azimuth pattern is widened to be compatible with the wide Rx pattern while keeping the transmit power constant. This results in the two-way pattern depicted in figure 2 (left part) and in comparison to the original TS-X configuration to a reduction in Tx gain by $3 \mathrm{~dB}$, i.e. $6 \mathrm{~dB}$ reduction in the two-way gain. The Configuration $\mathbf{B}$ is shown in figure 2 (right part) which assumes an unmodified Tx pattern together with the wide $\mathrm{Rx}$ pattern. This configuration results in a reduction in two-way gain of only $-3 \mathrm{~dB}$ but in a smaller overall beam width.

The two-way 6-dB azimuth beamwidth of TerraSAR-X is $0.33^{\circ}$ in the standard modes with complete $\mathrm{Rx}$ antenna, $0.66^{\circ}$ for configuration $\mathrm{A}$ and $0.41^{\circ}$ for configuration $\mathrm{B}$. In terms of azimuth bandwidth these values correspond to about 2800 , 5600 and $3500 \mathrm{~Hz}$, respectively. These different configurations are interesting when discussing the azimuth ambiguities later. 


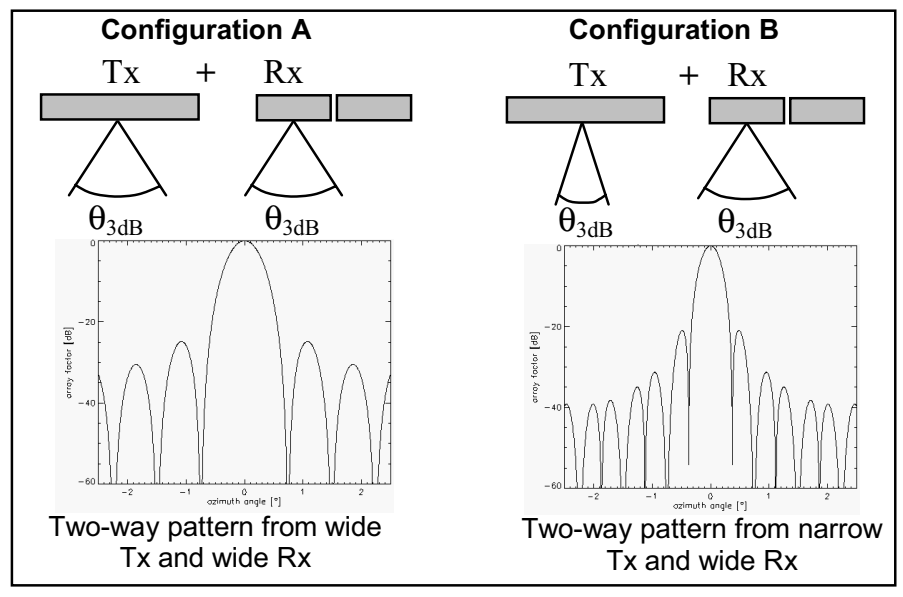

Figure 2. Two-way antenna pattern of the two configurations

\section{ALONG TRACK INTERFEROMETRY (ATI)}

With ATI the radial velocity component of moving objects can be measured. The sensitivity of the instrument mainly depends on the radar carrier frequency and the effective time lag between the two measurements carried out with two antennas and receiver chains. These parameters have to be adapted to the velocity range of the objects of interest. High speed objects like cars need only a very short time lag and the two antennas have to be separated my some meters. The measurement accuracy which can be achieved with TS-X has been estimated in [7].

Slow moving ocean currents would ideally require much longer time lags but it has been shown in [8] that even with the simple TS-X DRA mode many interesting ocean current applications can be performed.

\section{STRIPMAP RESOLUTION ENHANCEMENT}

A geometrical resolution enhancement of the TS-X Stripmap mode can be obtained by the DRA. In general a reduction of the antenna length in azimuth widens the antenna pattern. This results in a longer target illumination time, thus in an increase of the Doppler bandwidth and an improvement of the azimuth resolution. The drawback is that increasing the beamwidth demands also an increase of the PRF. Another problem is the reduction in SNR. In order to overcome these problems, two methods have been investigated.

Figure 3 shows the principles of the two methods. The left draft shows the TerraSAR-X antenna (with a length of $4.8 \mathrm{~m}$ ) at two succeeding azimuth positions. The phase center in Tx is in the center of the overall antenna and symbolized by the center spot. The phase centers of the two receiving antennas are the two outer spots. They are separated by $2.4 \mathrm{~m}$. The resulting two-way phase centers are at the position of the crosses. On the right draft, only the two-way phase centers are tagged.

\section{A. Method 1: Combining Rx Raw Data}

The signal of a reflected pulse is received with both antenna parts simultaneously. Thus, two spatial samples in azimuth direction are provided simultaneously, as shown by the crosses in figure 3 on the left. The PRF is adjusted in such a way that the

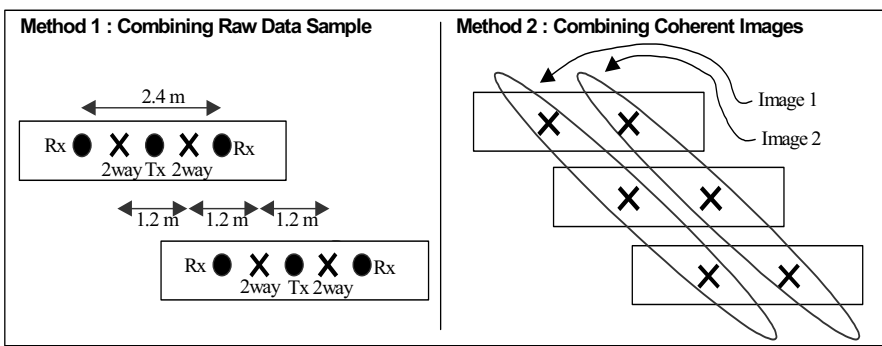

Figure 3. Resolution enhancement by combining Raw Data Sample (left) and Coherent Processed Images (right)

the next two samples received from the next reflected pulse are positioned subsequently to the first two samples. This results in a sequence of spatially equidistant samples. In this way, a halving of the sample spacing in azimuth is achieved, corresponding to a doubling of the overall PRF. The overall PRF is the PRF in the combined raw data.

The transmit PRF cannot be chosen arbitrarily but must be calculated as the quotient of the satellite velocity and the two way phase center distance times two. This can be seen in figure 3. Assuming a velocity of $7600 \mathrm{~m} / \mathrm{s}$ and a receiving phase center distance of $2.4 \mathrm{~m}$, the transmit PRF should be $3166 \mathrm{~Hz}$ and the overall PRF results in $6332 \mathrm{~Hz}$. This PRF is sufficient for proper sampling of the Doppler bandwidth of about $5600 \mathrm{~Hz}$ (Configuration $\mathrm{A}$ in section II). Due to timing constraints, the transmit PRF cannot be always exactly $3166 \mathrm{~Hz}$, but small deviations from this value do not reduce the overall PRF too much.

The advantage of this method is that the PRF is only virtually doubled and the original swath width of the stripmap mode can be maintained. The azimuth resolution can be doubled but the SNR will be deteriorated corresponding to a $6 \mathrm{db}$ gain loss of the antenna. Therefore this mode will be useful for applications which require high resolution and wide swath but tolerate an increased image noise.

\section{B. Method 2: Combining Coherently Processed Images}

If the maintenance of the swath width is not an issue the possibility of increasing the instrument PRF can be considered. The TerraSAR-X instrument allows for a PRF up to $6500 \mathrm{~Hz}$ and a proper sampling of the increased Doppler bandwidth of about $5600 \mathrm{~Hz}$ for Configuration A can be ensured.

In order to adapt the receive channel(s) to the increased $\mathrm{Tx}$ beamwidth either the complete receive antenna beam may be broadened or (like in Configuration B) the halfed antenna is used. The second channel can also be recorded and processed and used for ATI as well as for noise reduction. As shown in figure 3 on the right this means processing of one image from the front antenna and one from the rear. By coherent addition of the two complex images, $3 \mathrm{~dB}$ of SNR can be recovered while the azimuth resolution is as high as in one single image.

This mode is appropriate for ATI applications which require a doubled azimuth resolution in the stripmap mode and tolerate an increased SNR and a reduced swath width. For SAR imaging the SNR can be improved by combining the two channels. 


\section{FULL POLARIMETRIC MODE}

The TerraSAR-X instrument can toggle the polarisation switch within the T/R-Module in order to operate $\mathrm{H}$ and $\mathrm{V}$ polarisation alternately. This can be done in transmit and receive operation. Several strategies have been discussed to obtain two polarizations in parallel, i.e. interleaved pulses and interleaved bursts in ScanSAR or Spotlight manner [2].

In the DRA mode it is possible to obtain the full polarization matrix. As shown in figure 4, the complete antenna transmits alternating $\mathrm{H}$ - and V-polarized pulses. The backscattered signal is received simultaneously by both partitions of the antenna, one in H-polarization and the other in V-polarization.

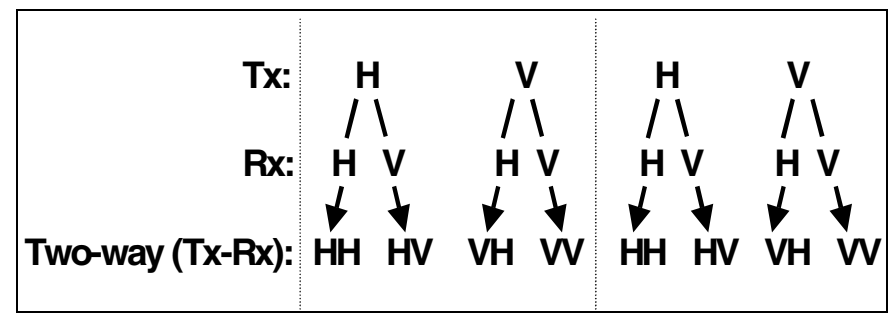

Figure 4. Polarization scheme for full polarimetric mode exploiting the DRA

Since the polarization in transmit is toggled between $\mathrm{H}$ and $\mathrm{V}$, the maximum available PRF in each of the two receiving chains is $3250 \mathrm{~Hz}$, half of the maximum TerraSAR-X PRF of $6500 \mathrm{~Hz}$. Since the azimuth bandwidth is increased in DRA mode (see section II), the azimuth ambiguities are too high and have to be reduced. This can be done by reduction of the processed azimuth bandwidth and by selection of the proper antenna configuration, A or B from section II.

In figure 5, the azimuth ambiguity ratio for TerraSAR-X is calculated for a processed bandwidth of $1300 \mathrm{~Hz}(\approx 5.2 \mathrm{~m}$ resolution). The two plots on the left show the ambiguous azimuth frequencies in black and the non-ambiguous processed frequencies in gray for a PRF of $3250 \mathrm{~Hz}$. The very left plot is for configuration $\mathrm{B}$ and the middle plot for configuration $\mathrm{A}$.

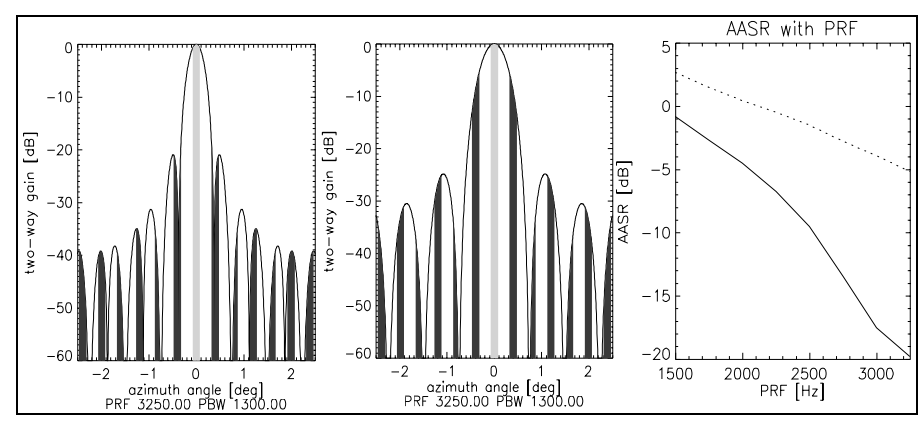

Figure 5. Azimuth ambiguities for configurations A (middle) and B (left) of section II with $1300 \mathrm{~Hz}$ processed bandwidth and $3250 \mathrm{~Hz}$ PRF. Azimuth ambiguity ratio as a function of PRF (right)

Figure 5 shows on the right the azimuth ambiguity ratio as a function of effective PRF in one polarimetric channel for configuration $\mathrm{A}$ in dotted style and for B in continuous style. It can be seen that configuration B provides much better azimuth ambiguity performance. The azimuth ambiguity ratio is better than $15 \mathrm{~dB}$ for PRFs higher than $2700 \mathrm{~Hz}$ in each polarimetric channel (instrument Tx PRF of $5400 \mathrm{~Hz}$ ).

\section{DISCUSSION}

The utilization of the Dual Receive Antenna Mode of TerraSAR-X has been presented with respect to three interesting new applications enabled by this mode. Since the instrument was originally not designed for DRA, the performance of the new applications is not optimized. However, taking advantage of the high instrument flexibility, reasonable performance for the new experimental modes along track interferometry, geometric stripmap resolution enhancement and full polarimetric mode can be achieved.

\section{ACKNOWLEDGMENT}

The authors would like to thank Christoph Heer, Martin Suess and Sebastian Riegger from the ASTRIUM GmbH in Friedrichshafen for useful discussions on the DRA concept. Furthermore the excellent co-operation between the industry and agency project managers enabled the introduction of the DRA mode into the TerraSAR-X design at a very late design phase.

Alberto Moreira had the idea to coherently combine two images for SNR improvement. A deeper statistical discussion on SNR with Adele Fusco was very helpful in this context.

\section{REFERENCES}

[1] M. Suess, S. Riegger, W. Pitz, R. Werninghaus: TERRASAR-X - Design and Performance, Proc. of EUSAR2002, Köln, Germany.

[2] J. Mittermayer, V. Alberga, S. Buckreuß, S. Riegger: TerraSAR-X: Predicted Performance, Proc. of Spie 2002.

[3] SRTM home page: http://www.dfd.dlr.de/srtm/neu/neu.htm

[4] H. Runge, M. Eineder, H. Breit, P. Flament, R. Romeiser, Measurement Of Tidal Current Fields With SRTM Along Track Interferometry, EGSAGU-EUG Meeting Nice, France, 6-11 April 2003

[5] R. Romeiser, H. Breit, M. Eineder, H. Runge, P. Flament, K. de Jong, J. Vogelzang: Validation of SRTM-Derived Surface Currents Off The Durch Coast By Numerical Circulation Model Results, IGARSS 2003, Toulouse, France, (in these proceedings)

[6] H. Breit, M. Eineder, J. Holzner, H. Runge, R. Bamler, Traffic Monitoring Using SRTM Along-Track Interferometry, IGARSS 2003, Toulouse, France, (in these proceedings)

[7] M. Eineder, S. Lehner: Discussion of TerraSAR-X Potential for Object Motion Measurements using Along Track Interferometry (ATI), Internal Technical Note TS-MF-POMMATI, 7.8.2002.

[8] R. Romeiser, H. Breit, M. Eineder, H. Runge, P. Flament, K. de Jong, J. Vogelzang, On The Suitability Of TerraSAR-X Split Antenna Mode For Current Measurements By Along Track Interferometry, IGARSS 2003, Toulouse, France, (in these proceedings) 